\title{
Primary pancreatic lymphoma: diagnostic pitfall of an uncommon medical entity mimicking operable pancreatic carcinoma: case report and review of literature
}

\author{
Dinesh Manchikanti, Manisha Aggarwal, Janitta Kundaikar, Shaji Thomas*, Ashish Arsia, \\ Rahul Pusuluri, Kusum Meena, Sanjay Kumar
}

Department of Surgery, Lady Hardinge Medical College and Dr. RML Hospital, New Delhi, India

Received: 12 May 2021

Accepted: 11 June 2021

*Correspondence:

Dr. Shaji Thomas,

E-mail: drshajithomas@yahoo.com

Copyright: () the author(s), publisher and licensee Medip Academy. This is an open-access article distributed under the terms of the Creative Commons Attribution Non-Commercial License, which permits unrestricted non-commercial use, distribution, and reproduction in any medium, provided the original work is properly cited.

\begin{abstract}
Almost all pancreatic neoplasms, including the commonest pancreatic ductal adenocarcinoma (PDAC) are derived from pancreatic epithelial components. Primary pancreatic lymphoma (PPL) is rare, accounting for less than $0.5 \%$ of all primary pancreatic tumours and presents a diagnostic and therapeutic challenge owing to their rarity, difficult access, and clinical and imaging features, which can mimic PDAC. A 26-year-old man presented with pain abdomen, jaundice, anorexia and weight loss for two months, along with two episodes of hematemesis and melena. Examination revealed an icteric patient with no palpable abdominal lump. His had a serum bilirubin of $13.6 \mathrm{mg} / \mathrm{dl}$. His ultrasound abdomen, CT scan and MRCP showed a mass in the head and uncinate process of the pancreas with circumferential nodular thickening in second part of duodenum. Side viewing endoscopy was suggestive of carcinoma head of pancreas with duodenal infiltration, for which endoscopic biopsy was taken which revealed non-Hodgkin's lymphoma B cell type. A PET CT showed a metabolically active mass lesion in the head of pancreas, with no other focus of disease anywhere else, suggestive of primary pancreatic lymphoma. This patient was referred to Medical oncology and started on chemotherapy. Lymphoma should be considered when a large, homogeneous, hypoenhancing mass is encountered in the pancreatic head, and with bulky lymphadenopathy. Preoperative tissue diagnosis such as fine needle aspiration cytology with or without flow cytometry and/or laparoscopy/endoscopy guided tissue biopsy may be necessary. It is crucial to differentiate PPL from pancreatic adenocarcinoma since their treatment is primarily medical and prognosis differs considerably.
\end{abstract}

Keywords: Primary pancreatic lymphoma, Non-Hodgkin lymphoma, Obstructive jaundice, Pancreas, Adenocarcinoma pancreas

\section{INTRODUCTION}

Almost all neoplasms of the pancreas are derived from pancreatic epithelial components, including the most common pancreatic mass, pancreatic ductal adenocarcinoma (PDAC). Nonepithelial neoplasms comprise only $1 \%-2 \%$ of all pancreatic neoplasms. The most common malignant or potentially malignant nonepithelial pancreatic tumors are of mesenchymal origin and include soft-tissue sarcomas, solitary fibrous tumor, and inflammatory myofibroblastic tumor.1 Hematopoietic tumors, including lymphomas, can manifest as isolated pancreatic involvement or secondarily involve the pancreas as widespread disease. ${ }^{1}$

Most cases of pancreatic non-Hodgkin lymphoma are part of a disseminated disease. Primary pancreatic lymphoma is rare and accounts for less than $0.5 \%$ of all primary pancreatic tumours. ${ }^{1,2}$ Primary pancreatic lymphoma represents a diagnostic and therapeutic challenge owing to 
their rarity, difficult anatomic location to access, and clinical and imaging features, which can mimic those of PDAC. However, it is important to consider these diagnoses and, when possible, differentiate primary pancreatic lymphoma from PDAC because they have vastly different treatments and prognosis.

\section{CASE REPORT}

A 26-year-old man presented to our outpatient department with complaints of pain in the upper abdomen for last three months and progressive jaundice for the last two months. He had a history of two episodes of hematemesis followed by melena in the last two weeks. He also had anorexia and significant weight loss. Examination revealed a deeply icteric patient with no palpable abdominal lump. His biochemical parameters showed a serum bilirubin of 13.6 $\mathrm{mg} / \mathrm{dl}$ with a direct bilirubin of $9.1 \mathrm{mg} / \mathrm{dl}$ along with elevated serum alkaline phosphate.

An ultrasound of the abdomen revealed a heterogeneous hypoechoic mass lesion in the head of the pancreas with the CBD dilated up to $9 \mathrm{~mm}$ with a distended gallbladder. MRCP and CECT abdomen showed a mass in the head and uncinate process of the pancreas with circumferential nodular thickening in second part of duodenum with encasement of superior mesenteric vein and portal vein (Figure 1). Side viewing endoscopy and ERCP were suggestive of carcinoma head of pancreas with duodenal infiltration, for which CBD stenting done, along with biopsy from the site of duodenal infiltration.

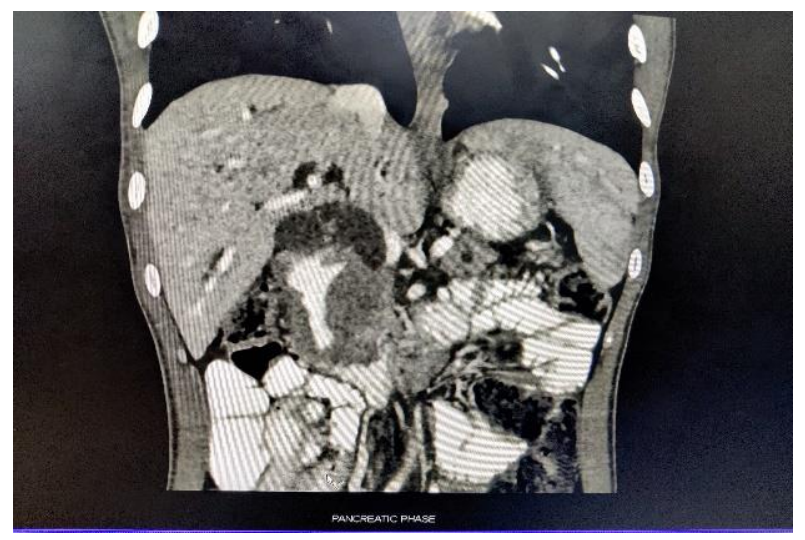

Figure 1: CT scan of the abdomen showing mass lesion in the head and uncinate process of pancreas with involvement of adjacent duodenum.

Endoscopic biopsy revealed non-Hodgkin's lymphoma B cell type (maltoma), CD 20 +ve and CD 3 -ve. CA 19.9, CEA and beta 2 microglobulin were within normal limits. LDH levels were mildly elevated and a PET CT showed a metabolically active ill-defined heterogeneous density mass lesion in the head and uncinate process of pancreas, with no other focus of disease anywhere else in the body, suggestive of primary pancreatic lymphoma (Figure 2). This patient was referred to Medical oncology and has been started on chemotherapy.

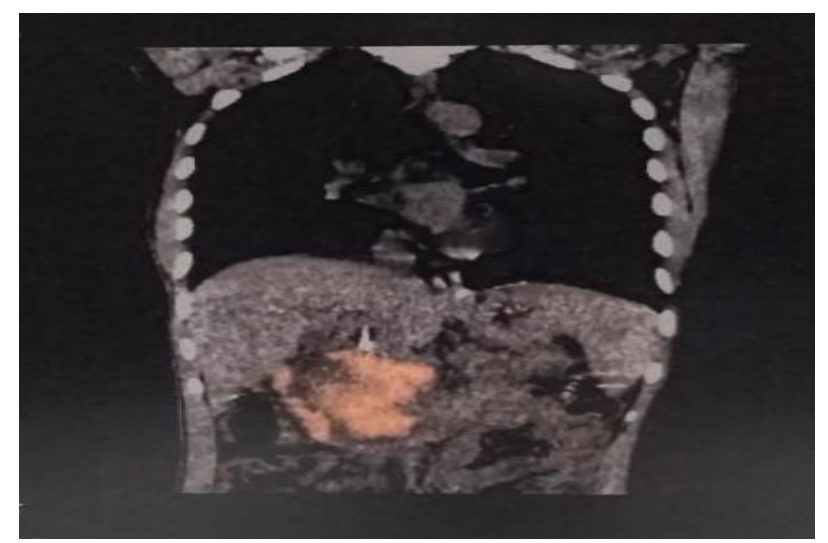

Figure 2: PET CT showing a metabolically active ill defined heterogeneous density mass lesion in the head and uncinate process of pancreas, with no other focus

of disease anywhere else in the body, suggestive of primary pancreatic lymphoma.

\section{DISCUSSION}

The most common pancreatic malignancy is pancreatic ductal adenocarcinoma (PDAC), an invasive malignant epithelial neoplasm with glandular (exocrine) differentiation. Nonepithelial neoplasms, including lymphomas, comprise only $1 \%-2 \%$ of all pancreatic neoplasms. ${ }^{1}$

Table 1: LUGANO staging for primary GI lymphoma. $^{7}$

\begin{tabular}{|c|c|}
\hline Stage & Extent of lymphoma \\
\hline I & $\begin{array}{l}\text { Confined to GI tract (single primary, or } \\
\text { multiple non-contiguous lesions) }\end{array}$ \\
\hline II & $\begin{array}{l}\text { Extending into abdomen from primary GI } \\
\text { site } \\
\mathrm{II}_{1}=\text { local nodal involvement } \\
\mathrm{II}_{2}=\text { distant nodal involvement }\end{array}$ \\
\hline II E & $\begin{array}{l}\text { Penetration of serosa to involve adjacent } \\
\text { organ or tissues } \\
\text { Specify site of involvement, e.g. IIE } \\
\text { (pancreas) } \\
\text { If both nodal involvement and involvement } \\
\text { of adjacent organs, denote stage using both } \\
\text { a subscript ( } 1 \text { or } 2 \text { ) and E, e.g. } \mathrm{II}_{1} \mathrm{E} \\
\text { (pancreas) }\end{array}$ \\
\hline IV & $\begin{array}{l}\text { Disseminated extra-nodal involvement or } \\
\text { concomitant supra-diaphragmatic nodal } \\
\text { involvement }\end{array}$ \\
\hline
\end{tabular}

Gastrointestinal lymphomas constitute $10-15 \%$ of all nonHodgkin lymphomas and $30-40 \%$ of all extranodal lymphomas. ${ }^{3,4}$ Gastrointestinal non-Hodgkin lymphoma (NHL) usually involves the stomach and the small bowel. Rarely, it can also present as a pancreatic mass. Primary G.I lymphoma are defined as lymphomas predominantly involving any section of GI tract from oropharynx to rectum where the disease typically involves a single 
primary site or multiple sites within GI tract. ${ }^{5-7}$ (Table 1 for staging of primary gastrointestinal lymphoma).

Most commonly, lymphoma found in the pancreas is associated with widespread disease. When the bulk of the disease is localized to the pancreas, even in the presence of contiguous nodal involvement, it is considered primary pancreatic lymphoma (PPL). ${ }^{8}$ PPL is most commonly nonHodgkin lymphoma, and the B-cell subtype is much more common than the T-cell subtype and has a better prognosis. ${ }^{9,10}$ The most common histologic subtype is diffuse large B-cell lymphoma (DLBCL), followed by follicular lymphoma.

PPL accounts for $0.5 \%$ of all pancreatic tumors and $0.6 \%$ of extranodal lymphomas. ${ }^{8,11}$ Most patients are middleaged, male, and white. It is more commonly seen in immunocompromised patients. ${ }^{2,12}$ Although the pancreatic head is the most common location for PPL, obstructive jaundice is infrequently a symptom at presentation. ${ }^{2}$ The most common reported symptoms for patients with PPL are abdominal pain and weight loss. ${ }^{2}$

A study attempted to elaborate the distinguishing features between pancreatic carcinoma and pancreatic haematological malignancy. The study found that features more often seen in patients with hematologic malignancies includes young age at presentation, large tumor size, low preoperative CA19. ${ }^{9}$ level, history of a pre-existing haematological malignancy, presence of B symptoms, absence of jaundice or diabetes mellitus. ${ }^{13}$

As with other lymphomas, an elevated level of serum lactate dehydrogenase (LDH) is demonstrated in patients with PPL, although this is a poor prognostic factor. ${ }^{2}$ The serum level of tumor marker CA $19-9$ is usually elevated in patients with PDAC, but only $25 \%$ of patients with PPL appear to have an elevated CA 19-9 level.

Two morphological patterns of pancreatic lymphomas are described on imaging: a focal circumscribed mass or diffuse infiltration. At CT, the focal form of PPL can manifest as a bulky, well-circumscribed, focal, hypoattenuating mass that shows homogeneous hypoenhancement relative to the normal adjacent pancreas, with no or mild associated ductal dilatation or upstream atrophy. Necrosis and calcification are not seen. There may be infiltration around the peripancreatic vessels but no stenosis or occlusion. The infiltrative form causes diffuse enlargement of the pancreas, mimicking the appearance of acute pancreatitis. However, there will not be surrounding inflammation, and patients will not have clinical signs or symptoms of pancreatitis. Presence of infrarenal lymphadenopathy essentially rules out PDAC. ${ }^{1,14,15}$

The treatment and prognosis of PPL are vastly different from those of PDAC; therefore, accurate diagnosis is crucial. The standard of care for PPL is chemotherapy, which leads to high complete response rate and long disease-free survival similar to those of nodal nonHodgkin lymphoma. ${ }^{12}$

CHOP chemotherapy in combination with rituximab (RCHOP) is used in T-cell/histiocyte-rich B-cell lymphoma (T/HRBCL) as it is used in all CD20 positive nodal and extranodal lymphomas. ${ }^{16,17}$ Chemotherapy combined with radiotherapy may be useful for treatment. The role of surgery should be reserved for diagnostic or palliative intent.

Prognosis is far dependent on histopathology, being secondarily influenced by age, presence of extranodal disease, performance status and stage. Prolonged follow up is recommended to detect relapse. ${ }^{18}$

Lymphoma should be considered when a large, homogenous, hypoenhancing mass is encountered in the pancreatic head without pancreatic or biliary ductal dilatation or vascular stenosis or occlusion and in the presence of bulky lymphadenopathy (especially if it involves the infrarenal space). ${ }^{12}$ Correlation with clinical findings (absence of jaundice) and tumor markers, specifically elevated LDH level and normal levels of carcinoembryonic antigen and CA 19-9, further strengthens the likelihood of lymphoma over PDAC and warrants diagnostic biopsy, with requisite histologic and immunophenotypic studies.

Several features of PPL can help differentiate it from other pancreatic neoplastic and non neoplastic conditions, including PDAC. Such differentiation allows appropriate recommendations and patient treatment. Pre-operative tissue biopsy or cytological diagnosis would be necessary to prevent unwarranted surgery in suspected cases involving primary pancreatic lymphoma. ${ }^{19}$

\section{CONCLUSION}

Lymphoma should be considered when a large, homogeneous, hypoenhancing mass is encountered in the pancreatic head with or without pancreatic or biliary ductal dilatation or vascular stenosis or occlusion, and in the presence of bulky lymphadenopathy (especially if it involves the infrarenal space), while the presence of necrosis rules out lymphoma. Primary pancreatic lymphoma should be considered in the differential diagnosis of pancreatic tumours especially and an attempt to obtain preoperative tissue diagnosis such as fine needle aspiration (FNA) cytology with or without flow cytometry and/or Laparoscopy / endoscopy guided tissue biopsy may be necessary before deciding to proceed to radical surgery for suspected ductal adenocarcinoma. It is crucial to differentiate PPL from other space occupying lesions, such as pancreatic adenocarcinoma, and inflammatory ones, including acute or chronic pancreatitis, autoimmune diseases or pancreatic tuberculosis, since their treatment and prognosis may differ considerably. 
Funding: No funding sources

Conflict of interest: None declared

Ethical approval: Not required

\section{REFERENCES}

1. Manning MA, Paal EE, Srivastava A, Mortele KJ. Nonepithelial neoplasms of the pancreas, part 2: malignant tumors and tumors of uncertain malignant potential from the radiologic pathology archives. Radiographics. 2018;38(4):1047-72.

2. Mishra MV, Keith SW, Shen X, Bar Ad V, Champ CE, Biswas T. Primary pancreatic lymphoma: A population-based analysis using the seer program. Am J Clin Oncol. 2013;36(1):38-43.

3. Wu XC, Andrews P, Chen VW, Groves FD. Incidence of extranodal non-Hodgkin lymphomas among whites, blacks, and Asians/Pacific Islanders in the United States: Anatomic site and histology differences. Cancer Epidemiol. 2009;33(5):337-46.

4. d'Amore F, Brincker H, Gronbaek K, Thorling K, Pedersen M, Jensen MK, et al. NonHodgkin's lymphoma of the gastrointestinal tract: A populationbased analysis of incidence, geographic distribution, clinicopathologic presentation features, and prognosis. Danish Lymphoma Study Group. J Clin Oncol. 1994;12(8):1673-84.

5. Dawson IM, Cornes JS, Morson BC. Primary malignant lymphoid tumours of the intestinal tract. Report of 37 cases with a study of factors influencing prognosis. Br J Surg. 1961;49:80-9.

6. Lewin KJ, Ranchod M, Dorfman RF. Lymphomas of the gastrointestinal tract: a study of 117 cases presenting with gastrointestinal disease. Cancer. 1978;42:693.

7. Harada G, Felipe-Silva A, da Silva JGN. Early stage primary gastric diffuse large B-cell lymphoma in a young HIV-positive patient. Autops Case Rep. 2014;4(2):49-54.

8. Jaffe ES, Müller-Hermelink HK, Delabie J, Ko YH, Nakamura S. Lymphoma of the pancreas. In: Bosman FT, Carneiro F, Hruban RH, Theise ND, eds. WHO classification of tumours of the digestive system. 4th ed. Lyon, France: International Agency for Research on Cancer. 2010;332:60.

9. Nishimura R, Takakuwa T, Hoshida Y, Tsujimoto M, Aozasa K. Primary pancreatic lymphoma: clinicopathological analysis of 19 cases from Japan and review of the literature. Oncology. 200;60(4):322-9.
10. Grimison PS, Chin MT, Harrison ML, Goldstein D. Primary pancreatic lymphoma: pancreatic tumours that are potentially curable without resection-a retrospective review of four cases. BMC Cancer. 2006;6:117.

11. Freeman C, Berg JW, Cutler SJ. Occurrence and prognosis of extranodal lymphomas. Cancer. 1972;29(1):252-60.

12. Sadot E, Yahalom J, Do RKG, Teruya-Feldstein J, Allen PJ, Gönen M, et al. Clinical features and outcome of primary pancreatic lymphoma. Ann Surg Oncol 2015;22(4):1176-84.

13. Rock J, Bloomston M, Lozanski G, Frankel WL. The spectrum of hematologic malignancies involving the pancreas: Potential clinical mimics of pancreatic adenocarcinoma. Am J Clin Pathol. 2012;137(3):414-22.

14. Merkle EM, Bender GN, Brambs HJ. Imaging findings in pancreatic lymphoma: differential aspects. AJR Am J Roentgenol. 2000;174(3):671-5.

15. Chua SC, Rozalli FI, O'Connor SR. Imaging features of primary extranodal lymphomas. Clin Radiol. 2009;64(6):574-88.

16. Bouabdallah R, Mounier N, Guettier C, Molina T, Ribrag V, Thieblemont C, et al. T-cell/histiocyte-rich large B-cell lymphomas and classical diffuse large Bcell lymphomas have similar outcome after chemotherapy: a matched-control analysis. J Clin Oncol. 2003;21(7):1271-7.

17. Valera ET, Queiroz RG, Brassesco MS, Scrideli CA, Neves F, Tone LG. Anti-CD20 monoclonal antibody therapy and minimal residual disease status of $\mathrm{T}$ cell/histiocyte-rich large B cell non- Hodgkin lymphoma. Pediatr Blood Cancer. 2011;57(2):348-9.

18. International Non-Hodgkin's Lymphoma Prognostic Factors Project. A predictive model for aggressive non-Hodgkin's lymphoma. $\mathrm{N}$ Engl J Med. 1993;329(14):987-94.

19. Sallapan S, Abu Bakar NZ, Jarmin R, Masir N, Mohammed F. Primary follicular lymphoma of the pancreas: A rare tumour mimicking pancreatic carcinoma. Malays J Pathol. 2018;40(3):359-71.

Cite this article as: Manchikanti D, Aggarwal M, Kundaikar J, Thomas S, Arsia A, Pusuluri R et al. Primary pancreatic lymphoma: diagnostic pitfall of an uncommon medical entity mimicking operable pancreatic carcinoma - case report and review of literature Int Surg J 2021;8:2221-4. 